Bull. Mater. Sci., Vol. 1, No. 2, October 1979, pp. 95-98. (C). Printed in India.

\title{
Research in Magnetism at Indian Institute of Technology, Bombay
}

\author{
C M SRIVASTAVA \\ Department of Physics, Indian Institute of Technology, Bombay 400076
}

MS received 2 August 1979

\begin{abstract}
The experimental solid state physics group at the Indian Institute of Technology, Bombay, has been engaged in research on ferromagnetic resonance and relaxation, magnetic ordering and exchange phenomena in ferrimagnetic systems. A brief report on the facilities developed and the work done by the magnetism group is presented.
\end{abstract}

\section{Introduction}

The magnetism group of the Department of Physics, at Indian Institute of Technology (IIT), Bombay, consists of four faculty members and a number of scientists and technical staff. The department has low and high power ferromagnetic resonance spectrometers operating in the $10 \mathrm{GHz}$ range, vibrating sample magnetometer (indigenously developed), Mössbauer spectrometer, a set-up for the permeability spectrum studies in the $10 \mathrm{KHz}$ to $100 \mathrm{MHz}$ range, $\mathrm{BH}$ loop tracers for soft and hard magnetic materials and preparation facilities for magnetic oxides by normal sintering and hot press techniques. A brief description of the work done by the group in recent years is presented in this paper.

\section{Ferromagnetic resonance and relaxation}

The work in the area of ferromagnetic resonance started with the investigations on the internal fields at the dipoles in cavity perturbation techniques when samples are located at the base of a microwave cavity (Patni and Srivastava 1971). This led to the development of a technique by which the intrinsic $g_{\text {eff }}$ value could be obtained in magnetic insulators with accuracies better than $2 \%$. The effect of the eddy current and inhomogeneous demagnetisation fields in ferromagnetic resonance and relaxation was studied for the first time and a satisfactory explanation was offered for the size-dependence of the line-shift and line-broadening due to these two processes (Srivastava and Patni 1974; Srivastava et al 1977b). An exact solution technique for the determination of the dielectric and magnetic susceptibility of low loss microwave materials has been recently developed which helps to reduce substantially the high cost of the sophisticated instrumentation used presently in the techniques based on perturbation methods (Hanumanthrao and Patni 1978). 
The reliability and accuracy of the $g_{\text {eff }}$ values obtained by the technique developed here has helped in the identification of crystal field state of magnetic ions in the ordered state. It has been possible to show from the FMR $g_{\text {eff }}$ values that in strontium-doped rare earth orthocobaltites the $\mathrm{Co}^{3+}$ ions are in intermediate spin state and not in the high or low spin states (Bahadur et al 1979).

\section{High power spin wave instability}

During the past three years, the processes leading to the spin wave instabilities have been studied in garnets at high power levels. This required the development of a high power X-band bench operating at $9.39 \mathrm{GHz}$ with peak power of $80 \mathrm{~kW}$ and pulse width of $4 \mu \mathrm{sec}$. The measurements have been made in the parallel-pump configuration with the if and steady magnetic fields parallel to each other (Prakash et al 1978a). The dependence of the spin-wave line width on the wave vector and grain size has been studied for some substituted yttrium iron garnets.

\section{Magnetic relaxation at low frequencies}

The shape of the permeability spectrum has been studied as a function of magnetisation and grain size for a number of garnets with $4 \pi M_{8}$ varying from 90 to $1780 \mathrm{G}$ and grain size from $1 \mu$ to $20 \mu$. The investigation on samples with a wide range of $4 \pi M_{s}$ and grain size showed that the earlier proposition (Globus 1975) that resonance-type permeability spectra are due to the presence of inhomogeneous grains in the sample is incorrect. A new theory based on our observations (Prakash et al 1979) has been developed which explains the change from resonance to relaxed character of the permeability spectrum as the grain size and magnetisation are varied.

\section{Mössbauer spectroscopy}

Mössbauer spectroscopy has been used for the first time to observe the thermally excited domain wall oscillations. In the case of samples with high ratio of the relaxation to resonance frequencies for the domain wall motion, localised oscillalations within the potential well are executed by the domain wall as the temperature is increased (Srivastava et al 1976a). This gives rise to a superposition of two types of spectra, one hyperfine split six-finger pattern due to ions within the domains and the other a central quadrupole split doublet due to the ions lying in the path of the domain walls. Based on the above, we have explained the complicated behaviour of the observed relaxed type of Mössbauer spectra for ferrous zinc ferrites as a function of temperature (Srivastava et al 1976a, b, 1977a).

\section{Magnetic ordering}

In collaboration with the group at the Bhabha Atomic Research Centre, the neutron diffraction technique has been used to show that nickel-zinc ferrites have a canted Yafet Kittel type of spin ordering (Satya Murthy et al 1969). The magnetisation data and the $g_{\text {eff }}$ values agree with this type of magnetic structure. Sub- 
sequently our result was verified in other laboratories using Mössbauer spectroscopy. Similar canted structure has been concluded for the ferrous zinc system also on the basis of its equilibrium magnetic properties (Srivastava et al 1976b).

\section{Super-exchange interaction in spinel ferrites}

The exchange constants in ferrites obtained by Nèel (1948) on the basis of the two sublattice model are not in agreement with the existing theories of the superexchange. A new method of analysis of the temperature dependent magnetisation and paramagnetic susceptibility curves based on the three sublattice model has yielded (Srivastava et al 1979) exchange constants of spinel ferrites in agreement with Anderson's theory of super exchange. The most interesting result of this investigation is that the exchange energy is independent of the number of electrons present in the half or more than half-filled sub-shell as long as the route of the super-exchange interaction between the two magnetic electrons is not blocked.

\section{Spin glasses}

In collaboration with Prof. Girish Chandra of the Tata Institute of Fundamental Research, studies of some of the spin glass systcms like $\left(\mathrm{Au}_{1-}, \mathrm{Cu}_{w}\right)_{0} \mathrm{Fe}_{1-\mathrm{d}}$ have been undertaken (Chandra et al 1978).

\section{Hot press technique}

The hot press technique using silicon carbide dies for the preparation of garnets with controlled microstructure has been developed for the first time in the country (Prakash et al 1978b).

\section{Developmental projects}

The group has been working since 1973 on the development of polycrystalline yttrium iron garnets by normal and hot press sintering techniques for applications in the $L$ - to $X$-bands of microwave communication systems. We have recently undertaken two projects, on s sponsored by the Department of Science and Technology on single crystal and hot press MnZn ferrites and the other jointly with Advanced Centre of Materials Science, Indian Institute of Technology, Kanpur, on the development of magnetic bubble materials.

\section{References}

Bahadur D, Kollali S, Rao C N R, Patni M J and Srivastava C M 1979 J. Phys. Chem. Solids (in press)

Chandra Girish, Shaikh A W and Srivastava C M 1978 Nucl. Phys. Solid State Phys. Symp. Bombay (in press)

Globus A 1975 European Physical Society Conference on Soft Magnetic Materials (Cardiff) p. 2 Hanumanth Rao and Patni M J 1978 Nucl. Phys. Solid State Phys. Symp. Bombay (in press) Néel L 1948 Ann. Phys. 3137

Patni M J and Srivastava C M 1971 Ferrites (Tokyo: University Park Press) p. 551.

Prakash Om, Patni M J and Srivastava C M 1978a Presented at the tenth annual meeting of the Association of Magnetic Resonance Spectroscopists held at Ahmedabad, January 1. See also technical report no. RPC/YIG/MPHCG/55/78. 
Prakash Om, Patni M J and Srivastava C M 1978b Presented at the Golden Jubiliee session of the Indian Ceramic Society held at New Delhi, March 10-13, See also technical report no. RPC/YIG/PHPMG/ITM/63/79

Prakash Om, Aiyer R and. Srivastava C M 1979 Bull. Mater. Sci. 149

Satya Murthy N S, Natera M G, Youssef S I, Begum R J and Srivastava C M 1969 Phys. Rev. 181969

Srivastava C M and Patni M J 1974 J. Magn. Reson. 15389

Srivastava C M, Shringi S N, Srivastava R G and Nanadikar N G 1976a Phys. Rev. B14 2032

Srivastava C M, Shringi S N and Srivastava R G 1976b Phys. Rev. B14 2041

Srivastava C M, Shringi S N and Bommanvar A S 1977a J. Phys. 38 CI-43

Srivastava C M, Patni M J and Nanadikar N G 1977b J. Phys. 38 CI-267

Srivastava C M, Srinivasan G and Nanadikar N G 1979 Phys. Rev. B19 499 